\title{
Rehabilitating endodontically treated mandibular molar having inadequate coronal length with "Endocrown" - A neoteric clinical approach!
}

\author{
Dr. Rupal J Shah ${ }^{1}$,Dr. Sanjay Lagdive ${ }^{2}$,Dr. Vishal Verma ${ }^{3}$, \\ Dr. Satyaprakash Shah ${ }^{4}$,Dr. Shraddha Saini ${ }^{5}$. \\ ${ }^{I}$ (Professor \& Head of Department, Department of Prosthodontics \& Crown \& Bridge, Govt. Dental College \& \\ Hospital, Ahmedabad/ Gujarat University, India) \\ ${ }_{2}^{2}$ (Professor, Department of Prosthodontics \& Crown \& Bridge, Govt. Dental College \& Hospital, Ahmedabad/ \\ Gujarat University, India) \\ ${ }^{3,4,5}$ (Post Graduate Student, Department of Prosthodontics \& Crown \& Bridge, Govt. Dental College \& \\ Hospital, Ahmedabad/ Gujarat University, India)
}

\begin{abstract}
Preservation of the natural tooth structure or its strength reinforcement, as far as possible, should always be the prime conservative concern for a dental practitioner, than vigorous replacement of the missing teeth, as Muller De Van rightly quoted. Endodontic treatment often yields up sparse amount of tooth structure, with inadequate coronal bulk, when the carious invasion is extensive; thereby making the extra coronal coverage with a full contour crown, a challenging task for the prosthodontist for its rehabilitation. Hereby we have reported a case of a 26 years old male patient having undergone endodontic treatment in grossly decayed mandibular left first molar, and later on rehabilitated with an "Endocrown" by surpassing its limitation of inadequate crown height to maximal level of efficacy.
\end{abstract}

Keywords: Endocrown, Endodontic Treated Tooth, coronal rehabilitation.

\section{Introduction}

Post endodontic restoration of the treated tooth ought to preserve, protect and reinforce the existing tooth structure, while maintain the form, function, esthetics, and its solidarity to the maximal attainable levels. During the course of the endodontic intervention itself, one should stick along conservative lines, trying to achieve minimally invasive access openings and further preparations, with maximal tissue conservation being the epicenter of treatment. However more often than not, in the bid to eliminate carious enamel and dentinal tissues, the preparation forms need to sacrifice more of the tooth anatomy, leaving insufficient residual tooth structures as resultants. Thereby mechanically stabilizing the already weakened tooth-restoration complex turns out to be a daunting task for full crown coverage.

A number of options are available depending upon the clinical presentation. The choice of prosthesis, whether to opt for partial or full coverage ultimately depends on the structural integrity of the tooth, esthetic considerations, and protective requirements [1]. Traditionally post and core restorations were used for stabilizing remaining coronal tooth structures [2], however off lately, their principle of restoration has been questioned, due to their long term detrimental consequences such as root perforation, root weakness and failure of teeth restored with posts [3]. Technological advancements, especially in esthetic dentistry today provide us with the luxury to combat these shortcomings with restorations such as conservative inlays, onlays etc by utilizing the pulp chamber as a retentive medium, that has further inspired a novel breed of treatment modality in the form of acid etchable ceramics such as feldspathic, leucite, lithium disilicate based ceramics and endocrowns. Thus endocrown is a conservative treatment modality for restoration of endodontically treated teeth in which walls of pulp chamber can be used as a retentive resource.

The terminology "Endocrown" was coined given by Bindl and Mormann in 1999 [4].The endocrown is a total porcelain crown which is leuted to a de pulped posterior tooth using all resin cement. In this endocrown, macro mechanical retention is achieved by internal portion of pulp chamber \& to the cavity margin, and micro retention is obtained by adhesive cementation technique. In the narrated case report, conventional feldspathic porcelain fused to metal was fabricated on endodontically treated mandibular left first molar (36), due to economic constraints on the patient's part, but the protocol of tooth preparation for an Endocrown was sensibly applied. 


\section{Clinical Case Report}

A 24 years old male patient, reported to the Prosthodontics and Crown \& Bridge department, Government Dental College and Hospital, Ahmedabad for restoration of post endodontic treated lower tooth (ETT) lower left first molar. Intraoral examination revealed that tooth 36 was asymptomatic, ever since the endodontic treatment was concluded 1 month ago, without any pain \& discomfort. However the occlusal gingival height of the remaining crown structure was approximately $3 \mathrm{~mm}$ only (Figure 1). The radiographic findings revealed well obturated canals with no evident periapical changes. Taking into consideration the inadequate coronal height availability for full cast crown and the patient's insistence for an aesthetically pleasing crown, Porcelain fused to metal ENDOCROWN was proceeded with as the fixed prosthesis of choice, as it would provide good esthetics, better mechanical performance, and could be performed with less cost \& less clinical time. The entire procedure was explained to patient and written consent was taken from patient.

\section{Procedure}

Occlusal tooth reduction was begun with a green diamond wheel bur, which reduced occlusal surface at least $2 \mathrm{~mm}$ in the axial direction by orienting the bur along the major axis of tooth \& held parallel to occlusal surface. This ensured a flat surface and it determined the precise position of cervical margin. This form of occlusal reduction was termed as cervical "walk around" preparation. The cervical margin was deliberately kept supragingival by approximately $1 \mathrm{~mm}$. Axial preparation included only removal of undercuts in the access cavity which was carried by green heavy shoulder bur. Cervical band was polished with polishing bur which was kept around the entire surface of cervical band to produce flat $\&$ polished surface, thereby producing a cervical butt angle joint. The finished line appeared as a regular line with a sharp edge. This concluded with the extra coronal part of tooth preparation.

Next pulp chamber was opened, de roofed, and the coronal filling of miracle mixture was removed to the extent, that the root canal canal orifices sealed with gutta percha obturations could be reached. The advantage of the saddle like anatomy of the cavity floor was used up. The bur was oriented along the long axis of tooth \& total occlusal convergence of $7^{\circ}$ was used to shape coronal pulp chamber $\&$ access cavity continuous. The depth of the access cavity was kept, at least $3 \mathrm{~mm}$ (Figure 2). Care was taken not to remove too much tissue from the pulp chamber, to avoid excessive drilling of dentin. The pulpal floor was thus shaped up,and finished to obtain flat surface, perpendicular to the tooth axis. Gingival retraction cord 000 (Ultrapak, Ultradent) was applied and impression was made with Polyvinyl siloxane silicone (Aquasil LV, Dentsply DeTrey, Germany) of light and heavy consistency using a putty wash technique (Figures 3a \& 3b).

The conventional casting technique was employed for fabricating the metal coping part of the endo crown on the die cut master cast (Figures $4 \mathrm{a} \& 4 \mathrm{~b}$ ).. The ceramic build up was proceeded ahead with the layering or "paint on" technique incrementally. The finished \& polished endocrown obtained from the furnace after final firing cycle, was seated onto the master cast for verifying its marginal fit, accuracy and congruence with the tooth contours on cast before leuting intra orally (Figures 5). The Endo crown was cemented intra orally using GIC leuting agent (GC Fuji I) (Figure 6). The gross occlusal discrepancies were removed with the articulating paper strips before cementation. Post cementation radiographic view depicted harmonious seating of the crown. Follow up visits were scheduled at 24 hours, 48 hours, biweekly, 2 months and 4 months intervals. The patient expressed satisfaction in terms of masticatory efficiency and esthetics of the Endocrown, without any notable discomfort (Figure 7).

\section{Figures And Tables}

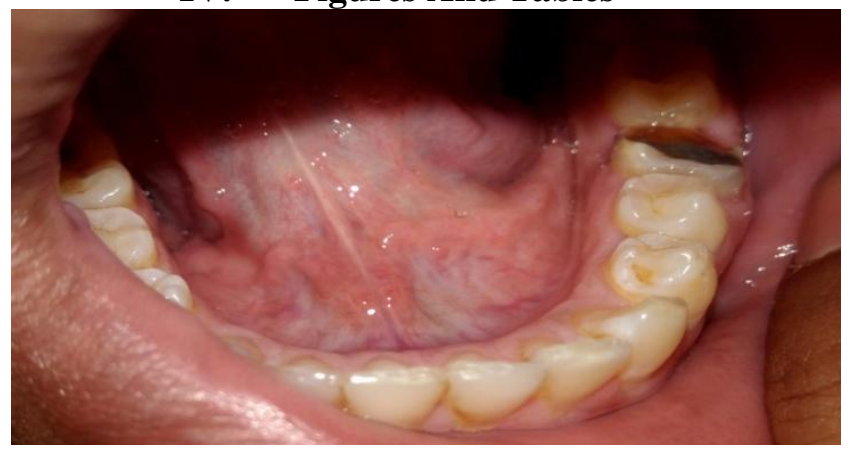

Figure 1: Preoperative view of Endodontically treated mandibular left first molar with inadequate crown height. 


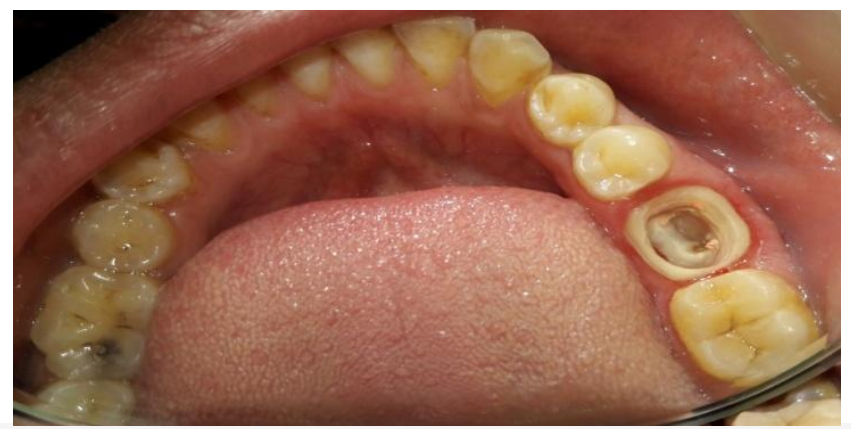

Figure 2: Completed Tooth preparation for an Endocrown (Cervical Buttjoint with a "WALK AROUND" preparation and internally $7^{0}$ converged walls)
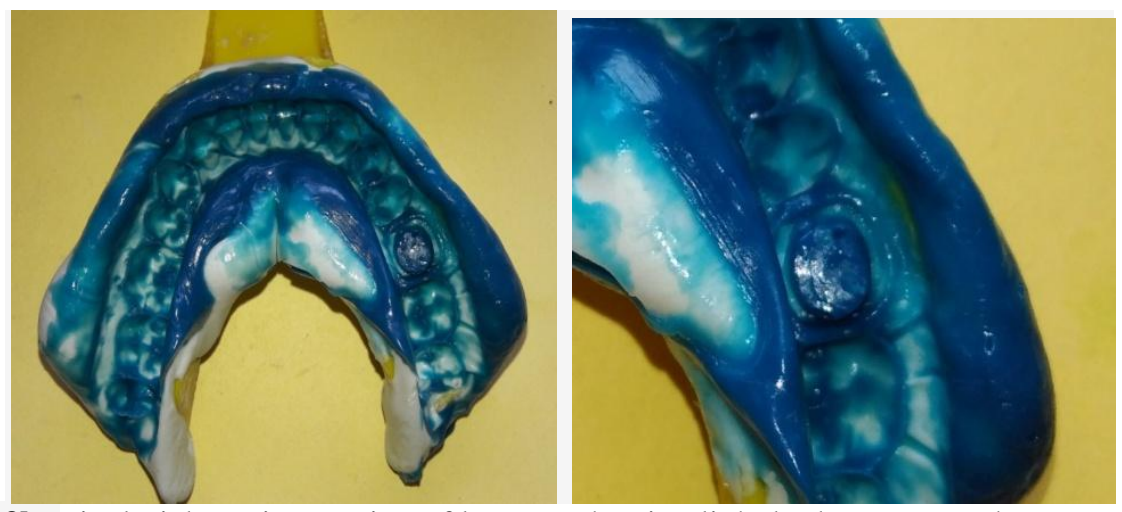

Figures 3a \& 3b: Final Pick Up impression of lower arch using light body Putty Wash Impression technique showing prepared 36 .

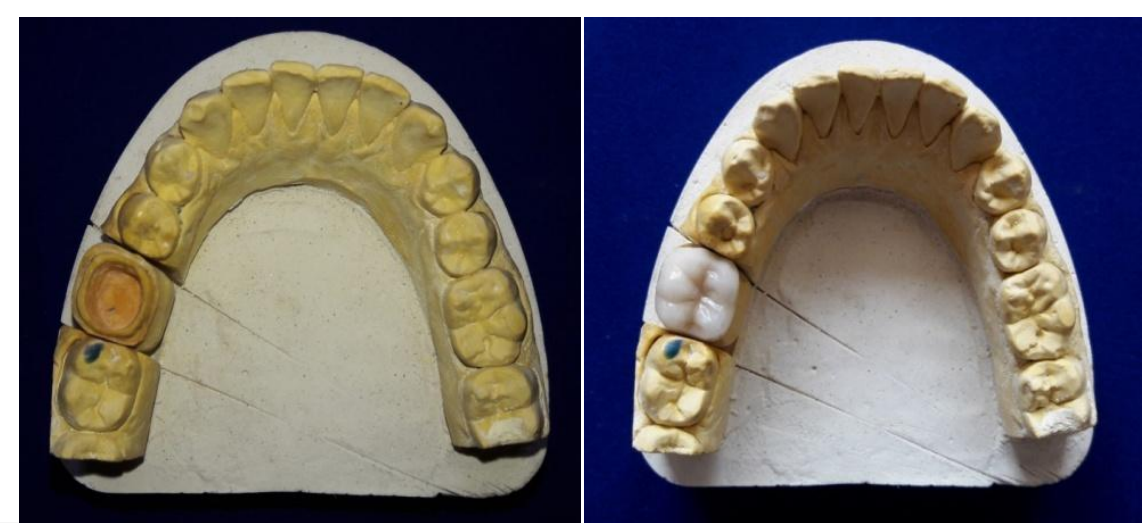

Figures 4a \& 4b: Die cut Master cast using Pindex system showing crown preparation in 36 for receiving Endo Crown

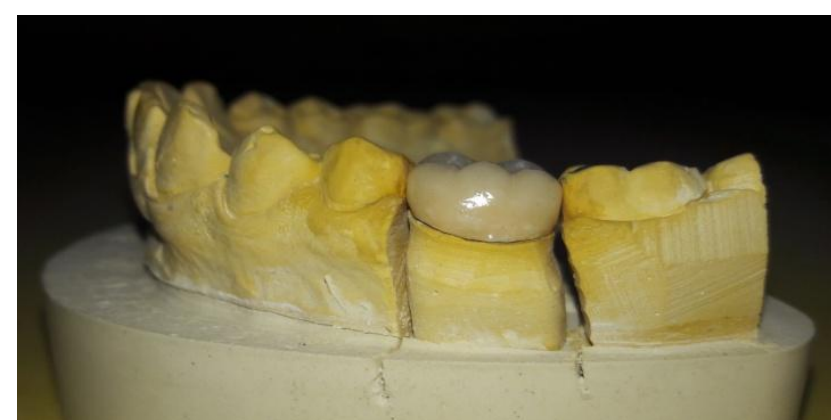

Figure 5: Margins of Endo Crown verified on master die cut cast before final leuting. 


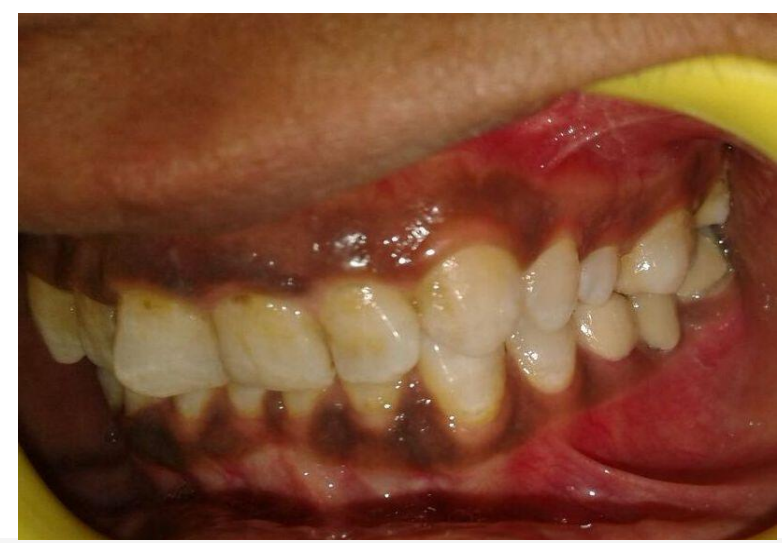

Figure 6: Buccal view of tooth 36 depicting the occlusion and imperceptible margins.

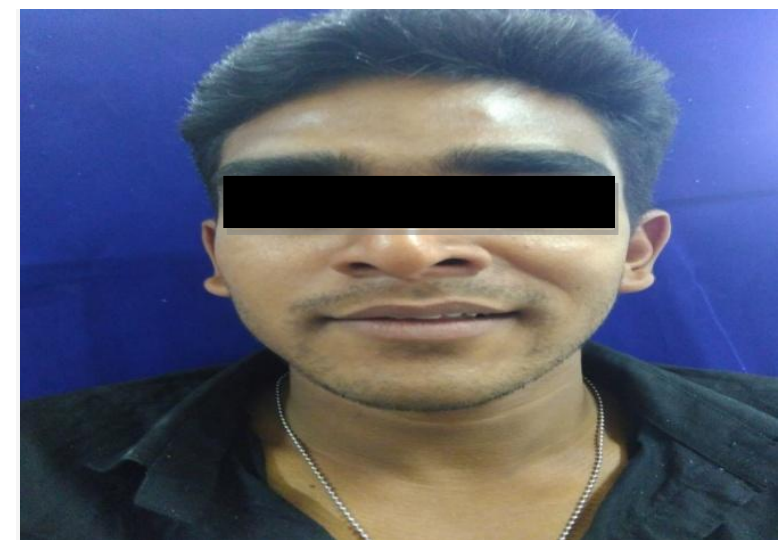

Figure 7: Post operative Smile on follow up visits.

\section{Discussion}

The case report entails a conservative treatment modality for a post endodontic treated mandibular left first molar tooth using Endocrown, wherein the residual coronal tooth structure ( $3 \mathrm{~mm}$ height) would have been inadequate for a conventional full cast crown.

Literary evidences clearly depict that the prosthesis of choice for reconstructing or restoring an endodontically treated tooth (ETT) is a tough call to make, and is largely governed by the voluminous amount of tooth structure remaining after the root canal therapy. A holistically sound, and long term sustainable restoration mandates reinforcement of the remaining healthy dental tissues, which can impart solidarity to tooth-restoration complex. In today's era esthetic \& adhesive dentistry, Endocrown serves as an able, conservative and feasible alternative to conventional post $\&$ core crowns, because it preserves root tissues and limits internal preparation of the pulp chamber to its anatomic shape.

It is a prime indication in cases of molars with obliterated, short, dilacerated or fragile roots and when limited inter occlusal space exists with the opposing dentition. However a carefully scrutinized clinical examination is necessary to see to it that the depth of pulp chamber should be atleast $3 \mathrm{~mm}$, and cervical enamel margin should be more than $2 \mathrm{~mm}$ wide [5]. For dimensions less than these, endocrowns are contraindicated! Besides, Endocrowns are indicated in cases where there are minimally subjected functional and lateral stresses on the tooth [6].

Different materials like feldspathic, glass ceramic, hybrid composite resin and newest CAD/CAM (computer aided design/ computer aided manufacturing) all-ceramic blocks can be used for fabrication of Endocrowns. In this case report however, a conventional Feldspathic porcelain fused to metal was fabricated, that was esthetically competent and economically much viable for the patient. Furthermore the preparation design is conservative compared to the traditional crown. Involvement of the biological width is minimal. In comparison to the post and core restorations, bonding surface offered by the pulpal chamber of the endocrown is often equal or even superior to that obtained from the bonding of a radicular post of $8 \mathrm{~mm}$ depth.

\section{Conclusion}

To put it in a nut shell, aspects such as meticulous planning, clever selection of restorative system, and the specifications \& precision in tooth preparation should be carefully considered before rehabilitating or restoring an endodontically treated tooth (ETT). Keeping ourselves abreast with the recent and innovative 
advancements of treatment modalities is the need of the hour. "Endocrown" is one such neoteric treatment option that combines the intra coronal form of restorations (inlay/onlay) with the extra coronal full coverage ones. It propagates bio integration and specifically addresses as an esthetic and functionally resounding clinical option for restoration of non-vital posterior tooth with reduced tooth structure.

\section{References}

[1]. K. Gulabivala. "Restoration of the root treated tooth," in Endodontics, C. J. R. Stock, K. Gulabivala, and R. Walker, Eds., pp. 279305, Elsevier, 3rd edition, 2004.

[2]. Rosabiacch, Robertatarkany Basting. The Endocrown: An Alternative Approach for Restoring Extensively Damaged Molars Journal of Esthetic and Restorative Dentistry 2013; 25(6); 383-90.

[3]. Dietschi D, Duc O, Krejci I, Sadan A. Biomechanical considerations for the restoration of endodonticallytreated teeth: A systematic review of the literature, part ii (evaluation of fatigue behavior, interfaces, and in vivo studies). Quintessence Int 2008;39(2):117129.

[4]. Bindl A, Mörmann WH. Clinical evaluation of adhesively placed Cerecendocrowns after 2 years - preliminary results. J Adhes Dent 1999; 1: 255-65.

[5]. Michel Fages, Bertrand Bennasar. The Endocrown: A Different Type of All Ceramic Reconstruction for Molars; J Can Dent Assoc 2013; 79: 140-41.

[6]. G.T.Rocca and B. Serge. Alternative treatments for restoration of non-vital teeth. Revue d'Odonto Stomatologie 2008; 37:259-272. 